\title{
Couple-Based HIV Prevention in the United States: Advantages, Gaps, and Future Directions
}

\author{
Nabila El-Bassel, DSW, * Louisa Gilbert, PhD, * Susan Witte, PhD, * Elwin Wu, PhD, * \\ Tim Hunt, MSW, and Robert H. Remien, PhD $†$
}

\begin{abstract}
This article presents an overview of couple-based HIV prevention research to date, advantages of using and core components of couple-based interventions, gaps in the current understanding of couple-based HIV prevention, status of dissemination research and the transportability of effective couple-based HIV prevention and treatment to real-world settings, and recommendations for future directions in couple-based prevention and treatment. Couple-based studies conducted among several populations-heterosexuals, men who have sex with men, and drug users - reported in the research literature were reviewed. Commonalities and limitations were noted in customary focus areas of the couple-based approaches: sexual and drug risk reduction, HIV testing behaviors, adherence to HIV treatment, and prevention of mother-to-child transmission. Couplebased intervention strategies have been rigorously tested and are a valuable addition to the arsenal of HIV prevention strategies. Immediate needs and opportunities include couple-based intervention strategies for prevention of HIV and other sexually transmitted infections among serodiscordant couples, couples who do not know their HIV status, and couples in whom both partners are HIV negative but at risk of HIV infection. There is a particular need to develop couple-based intervention strategies for men who have sex with men and for drug-involved couples.
\end{abstract}

Key Words: HIV/AIDS, couples, prevention

(J Acquir Immune Defic Syndr 2010;55:S98-S101)

\section{INTRODUCTION}

Although a 2008 surveillance report by the Centers for Disease Control and Prevention indicates that $86 \%$ of new HIV cases in the United States were attributed to sexual transmission, either through men who have sex with men (MSM; $54 \%$ ) or heterosexual contact (32\%), and although the proportion of new HIV cases attributed to sexual transmission has steadily increased since $2005,{ }^{1}$ most HIV prevention efforts in the United States have continued to focus on

From the *Social Intervention Group, School of Social Work, Columbia University, New York, NY; and †HIV Center for Clinical and Behavioral Studies, New York State Psychiatric Institute and Columbia University, New York, NY.

The authors have no funding or conflicts of interest to disclose.

Correspondence to: Nabila El-Bassel, DSW, Columbia University School of Social Work, 1255 Amsterdam Avenue, New York, NY 10027 (e-mail: ne5@columbia.edu).

Copyright (C) 2010 by Lippincott Williams \& Wilkins individual or group interventions, neglecting the critical role partners may play in transmission. ${ }^{2-4}$ Among both heterosexuals and MSM, sexual transmission of HIV occurs most frequently in the context of intimate relationships,,${ }^{5,6}$ a fact that underscores the need for couple-based HIV prevention (ie, involving both intimate sex partners in the HIV intervention).

A systematic review of couple-based HIV studies found that couple-based approaches are generally efficacious in promoting safer sex behaviors. ${ }^{7}$ Compared with individuallevel approaches, couple-based interventions are more efficacious in promoting HIV counseling and testing, ${ }^{7,8}$ supporting medication adherence among HIV-infected individuals in serodiscordant relationships, ${ }^{9}$ and improving adherence to treatment regimens for preventing motherto-child transmission. ${ }^{10,11}$

The few couple-based HIV interventions conducted in the United States have mostly focused on reducing unsafe sex or improving adherence to HIV medication. Most studies conducted internationally have focused on voluntary counseling and testing or prevention of mother-to-child transmission of HIV. ${ }^{7}$ All but 2 couple-based studies in the United States have been conducted with heterosexual men and women. ${ }^{7}$ This article presents the advantages and challenges of couple-based approaches, describes core components included in the couple-based studies, highlights the state of science of couple-based HIV research in the United States and the state of dissemination of these approaches to real-world settings, and discusses future directions that may improve couple-based HIV research in the United States.

\section{DISCUSSION}

\section{Advantages and Challenges of Couple-Based HIV Prevention}

The literature identifies several advantages of couplebased approaches. They provide an opportunity for the 2 members of the couple to recognize their mutual responsibility for protecting each other from HIV transmission and to work together to stay healthy. ${ }^{12-14}$ Couple-based approaches accentuate the relationship's context (ie, commitment, love, trust) and its connection to HIV acquisition, and then, they redirect attention to the value of the couple's relationship and the power of the dyad in behavior change. ${ }^{12-14} \mathrm{~A}$ safe environment is created that fosters discussion of sensitive or taboo topics (eg, sexual concurrency, power imbalances in the relationship, couple's sexual preferences, and sexual coercion). A couple-based modality allows the pair to learn together, 
in vivo with a third party (a facilitator), skills in couple communication, negotiation, problem solving, and couple goal setting as well as technical skills in condom use. Joint processing with the facilitator promotes accountability and increases commitment to change. ${ }^{12-15}$

\section{Core Components of Couple-Based HIV Prevention in the United States}

There is a notable heterogeneity in HIV intervention content in couple-based studies. ${ }^{7}$ Most studies explore the knowledge of HIV and sexually transmitted infections (STIs), technical skills in condom use, couple skills in communication and negotiation as well as problem solving and goal setting; couple power imbalances associated with sexual decision making ${ }^{7}$, and ways to promote and maintain a healthy relationship. Other content of HIV interventions addresses couple HIV counseling and testing, family planning, a review of cultural values to reinforce commitment to protect one's partner and community, and changing couples' peer norms regarding safer sex practices. ${ }^{712-15}$ This combination of couple-based skills and intervention content are illustrated in 3 studies conducted in the United States by the authors of this article.

The project Connect, with 217 heterosexual couples, was the first study funded by the National Institute of Mental Health of the National Institutes of Health to test a relationshipbased HIV/STI risk reduction intervention. The intervention, a series of 6 once-weekly sessions (delivered to couples in 1 study arm and to women alone in another), was found to be efficacious in increasing condom use, compared with the third study arm, the control - a single HIV/STI information session. ${ }^{12,13,15}$ The weekly sessions targeted the relationship context, and all exercises and homework assignments were geared toward the couple; all participants attending these sessions were asked to practice the communication, negotiation, and condom skills that they learned in the weekly sessions with their partners. Connect is currently being disseminated by the Centers for Disease Control and Prevention as a DEBI (diffusion of effective behavioral interventions) and tested for adoption by 80 New York State agencies. ${ }^{16}$

The project Eban, with 535 African American serodiscordant couples in 4 US cities, was recently completed. ${ }^{14,17}$ Couples assigned to the Eban couples risk reduction intervention compared with a health promotion comparison condition were more likely to increase condom use over the 12-month period. ${ }^{17}$

The project Connect with Pride, an adaptation of Connect that was targeted to African American MSM couples composed of at least 1 methamphetamine user, was recently piloted with 34 MSM dyads with promising results. ${ }^{18}$

Couple-based prevention research on drug-related HIV risks is emerging. Specific core components of drug-related interventions include syringe disinfection skills, triggers for drug use, gender imbalances in HIV risks associated with a couple's drug-using contexts, and improved access to harm reduction programs. ${ }^{19}$

Addressing treatment adherence within a couple has the potential advantage of not only improving the health of persons living with HIV but also reducing the risk of transmission within the pair by reducing the viral load of the infected partner and thus the risk of HIV transmission. ${ }^{9,20}$ Core components of interventions to promote adherence include enhancing a couple's commitment to maintain a steady supply of medications, helping to identify and resolve ongoing barriers to adherence, improving communication and mutual caretaking, promoting sexual risk reduction skills, and encouraging regular HIV testing for uninfected partners. In prevention of mother-to-child transmission interventions, couple sessions also focus on HIV testing for the male partner. ${ }^{10}$

\section{Gaps in the Science of Couple-Based Research}

Since the HIV epidemic began, only 6 couple-based studies have been conducted in the United States. ${ }^{9,15,17,18,21,22}$ Six of these focused on sexual risk reduction; 1 included both sexual risk reduction and adherence to antiretroviral therapy, where HIV prevention was incorporated into treatment and care. ${ }^{9}$ Two included MSM, ${ }^{9,18}$ and of these, 1 was conducted with MSM who use drugs. ${ }^{18}$ None was conducted solely with drug-using non-MSM populations. None has focused on primary prevention among HIV-uninfected couples who engage in HIV risks or among HIV-infected seroconcordant couples. ${ }^{7}$

Existing models vary in their definition of a "couple." In studies conducted by the authors, couples include ongoing dyadic heterosexual or same-sex relationships, which may include but are not limited to relationships between spouses, common-law spouses, intimate partners, lovers, and casual sexual partners. Most couple intervention models allow the index participant to identify his or her partner. Several have more stringent criteria, such as length of the relationship, level of commitment, and sexual orientation. ${ }^{15,17}$ Thus, key questions remain unanswered: To what extent are the findings generalizable? For whom are the interventions particularly efficacious?

Most couple-based studies are limited by 1 or more methodological drawbacks, including relatively small sample sizes, lack of a randomized control design, and/or the lack of biologically confirmed STIs as an outcome variable. ${ }^{7}$ None of the couple-based studies to date have been sufficiently powered to examine new STI and HIV infections as outcomes.

To date, few studies have examined whether couplelevel interventions are effective in reducing extradyadic sexual relationships among partners. This is an important outcome to consider, as partner concurrency has been found to increase HIV risk within a partner's sexual network and within the dyad. ${ }^{23}$ It is unclear whether existing couple-based HIV prevention approaches reduce HIV risk with extradyadic partners.

Couple-based HIV prevention approaches have used different modalities to deliver their intervention. The science of couple-based HIV prevention has yet to tell us which modality works better. Are interventions more effective when sessions are delivered to each couple individually, when the sessions bring a group of couples together, or when some sessions are delivered in small, single-gender groups? Future research should address the question of which is the most efficacious and/or cost-effective modality.

The particular mechanisms that lead to behavior change remain unspecified. To advance the science of HIV prevention intervention with couples, there is a need to identify mediators responsible for behavioral change. Moreover, greater attention 
must be given to the use of relationship-based theories that move beyond social cognitive theory, which has guided most couplebased studies. ${ }^{7}$ Statistical techniques using data collected from couples also need to be developed more thoroughly. Examples of areas to be targeted for development include the following: Is the unit of analysis the individual or the couple? What is a conservative way to handle discrepant reports from partners on conjoint behaviors?

A number of challenges need to be highlighted in the couple-based interventions. Although some evidence suggests that couple-based HIV prevention interventions may not work as well for couples experiencing severe conflict or intimate partner violence, ${ }^{24,25}$ research has yet to identify whether the effects of couple-based interventions are moderated by certain relationship characteristics (eg, length and type of relationship, relationship satisfaction level, history of intimate partner violence). Another challenge is that couple-based interventions require more clinical training and skills than individualbased interventions. Agency clinical staffs also tend to be more comfortable conducting individual interventions and may take more time to feel comfortable delivering couplebased sessions.

\section{Dissemination of Couple-Based Prevention Interventions}

Despite their demonstrated value in reducing risk behaviors and improving adherence to HIV medication, couple-based approaches are rarely employed in HIV service settings. Even partners who present and want to be treated together will likely not find such services. Most DEBI approaches that have been disseminated are delivered using individual or group modalities. ${ }^{16}$ Reasons for the limited dissemination and scaling up of couple-based HIV interventions to date may include common ideological preferences of staff and administrators for individual or group services, lack of structure to provide couple services within agencies, lack of access to evidence-based HIV interventions for couples, and lack of funding and staff training in couple-based modalities. Expanding the scope of dissemination and scaling up couplebased HIV interventions will require commitment on the part of government and donors to fund research on dissemination and implementation as well as training for providers in couplebased approaches.

\section{CONCLUSIONS}

Couple-based HIV interventions are in the early stages of development in the United States, compared with individual and group-level HIV interventions. Yet the advantages of couple-based approaches are evident. They can reduce drug and sexual behaviors that drive transmission of HIV and improve adherence to antiretroviral therapy and can, in turn, decrease risk of transmission. However, there remains a need for more research on couple-based approaches, especially for drug users and MSM and for seroconcordant couples.

The science of couple-based research can be advanced by addressing the gaps and challenges discussed in this article, particular assessing the impact of couple-based interventions on concurrent sexual relationships and biological outcomes of
STIs and HIV. More attention and resources must be given to the dissemination of evidence-based, couple-based prevention and treatment research into real-world settings. Advancing the science of couple-based research (eg, efficacy, effectiveness, and dissemination) has the potential to reduce HIV acquisition and transmission among vulnerable populations.

\section{REFERENCES}

1. Centers for Disease Control and Prevention. Diagnoses of HIV Infection and AIDS in the United States and Dependent Areas, 2008. HIV Surveillance Report. Vol 20. Atlanta, GA: Division of HIV/AIDS Prevention, National Center for HIV/AIDS, Viral Hepatitis, STD, and TB Prevention, Centers for Disease Control and Prevention; 2010. Available at: http://www.cdc.gov/hiv/surveillance/resources/reports/2008report/. Accessed August 12, 2010.

2. Herbst JH, Beeker C, Mathew A, et al. The effectiveness of individual-, group-, and community-level HIV behavioral risk-reduction interventions for adult men who have sex with men: a systematic review. Am J Prev Med. 2007;32:38-67. doi:10.1016/j.amepre.2006.12.006.

3. Coates TJ, Richter L, Caceres C. Behavioural strategies to reduce HIV transmission: how to make them work better. Lancet. 2008;372:669-684. PMCID: PMC2702246.

4. Johnson BT, Carey MP, Chaudoir SR, et al. Sexual risk reduction for persons living with HIV: research synthesis of randomized controlled trials, 1993 to 2004. J Acquir Immune Defic Syndr. 2006;41:642-650. PMCID: PMC2424183.

5. Sullivan PS, Salazar L, Buchbinder S, et al. Estimating the proportion of HIV transmissions from main sex partners among men who have sex with men in five US cities. AIDS. 2009;23:1153-1162.

6. Higgins JA, Hoffman S, Dworkin SL. Rethinking gender, heterosexual men, and women's vulnerability to HIV/AIDS. Am J Public Health. 2010; 100:435-445. doi:10.2105/AJPH.2009.159723.

7. Burton J, Darbes LA, Operario D. Couples-focused behavioral interventions for prevention of HIV: systematic review of the state of evidence. AIDS Behav. 2010;14:1-10. doi:10.1007/s10461-008-9471-4.

8. Desgrées-du-Loû A, Orne-Gliemann J. Couple-centred testing and counselling for HIV serodiscordant heterosexual couples in sub-Saharan Africa. Reprod Health Matters. 2008;16:151-161. doi:10.1016/S09688080(08)32407-0.

9. Remien RH, Stirratt MJ, Dolezal C, et al. Couple-focused support to improve HIV medication adherence: a randomized controlled trial. AIDS. 2005; 19:807-814.

10. Semrau K, Kuhn L, Vwalika C, et al. Women in couples antenatal HIV counseling and testing are not more likely to report adverse social events. AIDS. 2005;19:603.

11. Kiarie JN, Kreiss JK, Richardson BA, et al. Compliance with antiretroviral regimens to prevent prenatal HIV-1 transmission in Kenya. AIDS. 2003; 17:65-71.

12. El-Bassel N, Witte SS, Gilbert L, et al. HIV prevention for intimate couples: a relationship-based model. Fam Syst Health. 2001;19(4):379395. doi:10.1037/h0089467.

13. El-Bassel N, Witte SS, Gilbert L, et al. Long-term effects of an HIV/STI sexual risk reduction intervention for heterosexual couples. AIDS Behav. 2005;9:1-13. doi:10.1007/s10461-005-1677-0.

14. NIMH Multisite HIV Prevention Trial for African American Couples Group. Eban HIV/STD risk reduction intervention: conceptual basis and procedures. J Acquir Immune Defici Syndr. 2008;49:S15-S27.

15. El-Bassel N, Witte SS, Gilbert L, et al. The efficacy of a relationshipbased HIV/STD prevention program for heterosexual couples. $\mathrm{Am} \mathrm{J}$ Public Health. 2003;93:963-969. PMCID: PMC1447878.

16. Witte SS.Use of multimedia technologies to disseminate HIV prevention. Presented at: 2nd Annual NIH Conference on the Science of Dissemination and Implementation; January 28-29, 2009; Bethesda, MD. Abstract 43. Available at: http://conferences.thehillgroup.com/obssr/ di2008/01_Program\%20Book/01_DI09\%20FINAL\%20ProgramBook.pdf. Accessed August 17, 2010.

17. El-Bassel N, Jemmott JB, Landis JR, et al. NIMH Multisite Eban HIV/ STD Prevention Intervention for African American HIV serodiscordant couples: a cluster randomized trial. Arch Int Med. 2010;170:1594-1601. 
18. Wu E, El-Bassel N, McVinney L, et al. Adaptation of a couple-based HIV intervention for methamphetamine-involved African American men who have sex with men. Open AIDS J. 2010;4:123-131. PMCID: PMC2908928.

19. Gilbert L, El-Bassel N, Terlikbayeva A, et al. Couple-based HIV prevention for injecting drug users in Kazakhstan: a pilot intervention. J Prev Interv Community. 2010;38:162-176. doi:10.1080/10852351003640914.

20. Attia S, Egger M, Muller M, et al. Sexual transmission of HIV according to viral load and antiretroviral therapy: systematic review and meta-analysis. AIDS. 2009;23:1397-1404. doi:10.1097/QAD.0b013e32832b7dca.

21. Harvey SM, Bird ST, Galavoti C, et al. Relationship power, sexual decision making and condom use among women at risk for HIV/STDs. Women Health. 2002;36:69-84. doi:10.1300/J013v36n04_06.
22. Koniak-Griffin D, Lesser J, Henneman T. HIV prevention for Latino adolescent mothers and their partners. West $J$ Nurs Res. 2008;30:724-742. doi:10.1177/0193945907310490.

23. Halperin DT, Epstein H. Concurrent sexual partnerships help to explain Africa's high HIV prevalence: implications for prevention. Lancet. 2004; 364:4-6. doi:10.1016/S0140-6736(04)16606-3.

24. Greene K, Bogo M. The different faces of intimate violence: implications for assessment and treatment. J Mar Fam Ther. 2002;28: 455-466.

25. Jacobson NS, Gottman JM. When Men Batter Women: New Insights into Ending Abusive Relationships. New York, NY: Simon \& Schuster; 1988. 